\title{
A SURFACE MODELING PARADIGM For electromagnetic APPLICATIONS IN AEROSPACE STRUCTURES
}

RM Jha, SA Bokhari, V Sudhakar and PR Mahapatra Department of Aerospace Engineering

Indian Institute of Science

Bangalore, 560012 India

\section{ABSTRACT}

A systematic approach has been developed to model the surfaces encountered in aerospace engineering for EM applications. The basis of this modeling is the quadric canonical shapes which are the coordinate surfaces of the Eisenhart Coordinate Systems. The building blocks are visualized as sections of quadric cylinders and surfaces of revolution. These truncated quadrics can successfully model realistic aerospace structures which are termed as hybrid quadrics, of which the satellite launch vehicles are illustrative examples.

Introduction

The shapes of aerospace structures are primarily governed by aerodynamic considerations. The most common body shape is cylindrical, with a conical structure in the front, to effectively perform the fluid-piercing function. However, due to payload and other logistical considerations, innumerable other variants of the basic cone-cylinder configuration are encountered in practice. Similarly, blunting the nose tip regulates the shock and heat transfer characteristics. A combination of these requirements may lead to a shape resembling closely an cllipsoidit section or a paraboloid of revolution. Sirrilarly, most appendages to the flight vehicles, like the wings and external control surfaces may be closely approximated as hybrid surfaces consisting of segments with single curvatures. Such segments are typically derived from the circular, elliptic and parabolic cylinders. It is apparent that a vast majority of aerospace structures can be conveniently modeled as combinations of simpler quadric surfaces.

The work presented in this paper concerns the generalized solution of surface ray tracing problems associated with simple and hybrid surfaces of the type encountered in aerospace vehicles and satellites. The primary motivation for this work comes from the practical need to develop a corriprehensive theoretical approach to the high frequency analysis of antenna characteristics for the antennas located on realistic aerospace structures.

\section{Analytical Surface Generation and Surface Modeling}

The quadrics most commonly encountered among the aerospace shapes are the quadric cylinders (QUACYLS) and the quadric surfaces of revolution (QUASORS). The complete class of the quadric cylinders consist of circular, elliptic, hyperbolic and parabolic cylinders. Similarly the right circular cone, sphere, ellipsoid, and the hyperboloid and paraboloid of revolution constitute the mondegenerate class of quadric surfaces of revolution. These UUACYLS and UUASORS are the coordinate surfaces of the appropriate Eisenhart Coordinate Systems [1]. 
The Eisenhart Surfaces (i.e., the coinplete class of QUACYLS and QUASORs) form the basis of our surface modeling paradigm. However, it may be nuted that these surfaces are generally infinite or semi-infinite. Since, in practice, the surfaces are but finite, the building blocks of the aerospace shapes can be considered as the truncated sections of these quadrics and are identified as tQUACYLS and t-UUASORS.

Most aerospace shapes are however quite complex and can only be realized by a combination of finite number of these truncated quadrics. Such structures in our modeling have been identified as the hybrid quadrics (h-QUACYLs and $h$ QUASORs). Figure 1 show some of the illustrative examples of h-QUASORs.

One of the salient features of our paradigm is the retention of only convex building blocks, and an attempt to describe the surfaces while retaining its convexity. From the ray-theoretic point of view this has advantage that on such surfaces the surface ray geometric parameters can be readily obtained.

Yet another advantage of this model is realization of the shaping parameters corresponding to all the quadric in the Eisenhart Coordinate System in terms of the coordinate parameter held constant $[2,3]$. For example, the general equation of the generating parabola $x^{2}=b y=\left(a^{2}\right) y$, of a paraboloid or a parabolic cylinder, incorporates the real scalar constant a serving as a shaping parameter, by varying which parabolas of different sharpness/flatness can be generated.

In summary, the concept of modeling with sections of generatized quadrics is versatile and can accurately describe various complex, practical and realistic aerospace structures.

Although rrore complex surfaces than quadrics, such as, bicubics, have been considered for computer modeling application [4] and found to provide greater modeling flexibility because of their inherent ability to minimize or eliminate slope discontinuities, the mathematical complexity of these surfaces results in severe computational loads for complex scenes. It thus appears that the quadrics provide the best trade-off between the element simplicity and the model simplicity while describing complex scenes.

\section{Summary}

A surface modeling paradigm has been systematically constructed (Fig. 2). As a logical step after ray tracing over the canonical quadric shapes (i.e., individual QUACYLS and QUASORS), hybrid structures of practical interest are considered. Included in this class is the aircraft wing modeled as a hybrid QUACYL, for which the ray tracing parameters have been obtained in closed form [2]. Figure 2 also represents the mathematical analysis structure that has been formalized by these authors [5]. The paradigm also facilitates the treatment of the more complex case of the hybrid nondevelopable QUASORs such as a satellite launch vehicle modeled as a GPOR-cylinder combination. The ray geometric parameters have been obtained in the quasi-analytical form. 
REFERENCES

[1] P. Moon and D.E. Spencer, Field Theory Handbook. Berlin: Springer-Verlag, 1971.

[2] R.M. Jha, S.A. Bokhari, V. Sudhakar and P.R. Mahapatra, "Ray analysis on a class of hybrid cylindrical aircraft wings", Electronics Letters (GB), vol. 24, pp. 21-22, Jan. 1988.

[3] R.M. Jha, S.A. Bokhari, V. Sudhakar and P.R. Mahapatra, "Ray analysis on a class of nondevelopable satellite launch vehicles", Electronics Letters (GB), vol. 24, pp. 22-23, Jan. 1988.

[4] G.Y. Gardner, "Simulation of natural scenes using textured quadric surfaces", Computer Graphics, Proceedings of SIGGRAPH 84, vol. 18, no. 3, pp. 11-20, 1984.

[5] R.M. Jha, Surface Ray Tracing on Convex Quadrics with Applications to Mutual Coupling between Antennas on Aerospace Bodies, $\mathrm{Ph} D$ Dissertation. Subinitted to Department of Aerospace Engineering, Indian Institute of Science, Bangalore, India, Nov. 1988.
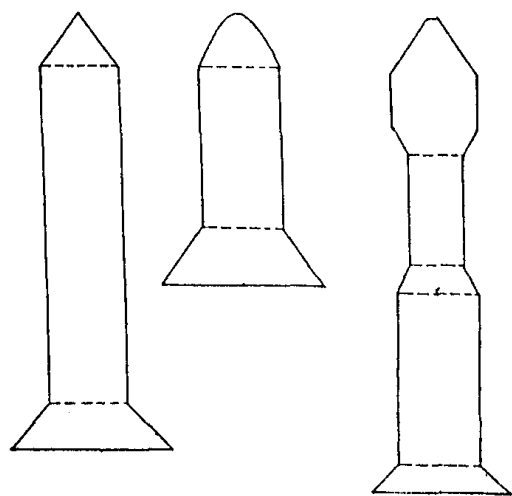

Fig. 1 Some examples of hybrid surfaces of revolution (h-QUASORs). 
1

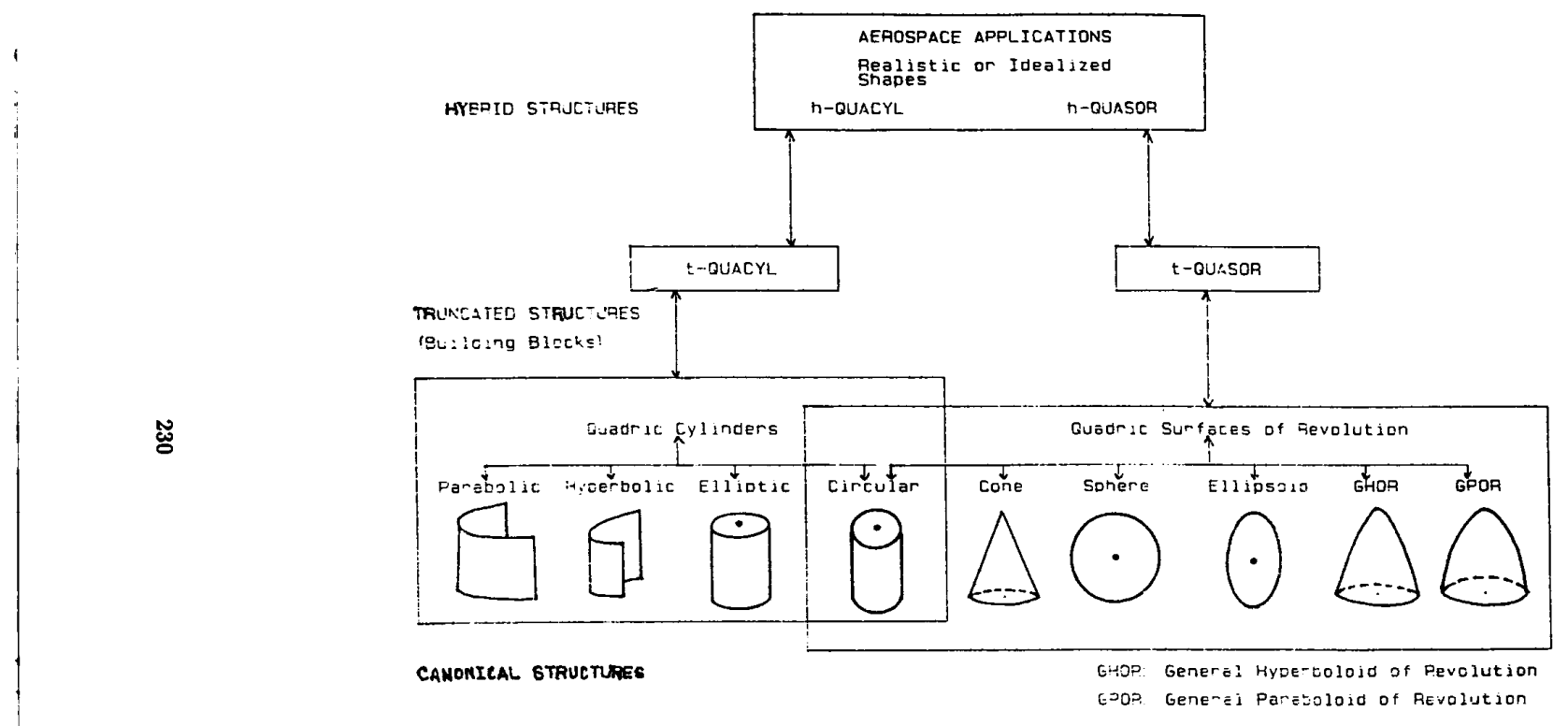

Fig. 2 Surface modeling paradigm.

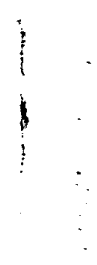

6

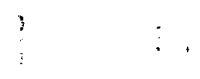

